\title{
ANSIEDAD PRE-COMPETITIVA Y ESTRATEGIAS DE AFRONTAMIENTO DEPORTIVO, EN DISCIPLINAS ACUÁTICAS INDIVIDUALES Y COLECTIVAS EN DEPORTISTAS JUVENILES DE ALTO NIVEL
}

\author{
PRE-COMPETITIVE ANXIETY AND SPORTS COPING STRATEGIES, IN \\ INDIVIDUAL AND COLLECTIVE WATER SPORTS IN HIGH LEVEL YOUTH \\ ATHLETES
}

\author{
Geraldy Sepúlveda-Páez*, Yasna Díaz-Karmelic, \& Rodrigo Ferrer-Urbina \\ Universidad de Tarapacá, \\ Arica - Chile.
}

Recibido junio de 2019Received June, 2019

Aceptado septiembre de 2019/Accepted September, 2019

\begin{abstract}
RESUMEN
Existe amplia evidencia de los efectos de la ansiedad precompetitiva (e.g. Bueno, Capdevila y Fernández- Castro, 2002; Caracuel y Jaenes, 2015) en las prácticas y pruebas deportivas, así como del rol protector de las estrategias de afrontamiento que emplean los deportistas (e.g., Soto, 2003). Sin embargo, debido a que las prácticas deportivas competitivas se diferencian, entre otras cosas, en su carácter colectivo o individual, tanto la ansiedad competitiva como las estrategias de afrontamiento involucradas pudiesen diferenciarse según dicha condición (e.g., Simón y Martens,1979). El presente estudio corresponde a una exploración sobre posibles efectos diferenciales del tipo de práctica sobre la ansiedad precompetitiva, las estrategias de afrontamiento y la relación entre ambas. El diseño fue transversal de tipo correlacional, en el cual se aplico el Inventario de Ansiedad Competitiva en el Deporte (CSAI-2) y el Cuestionario de Aproximación al Afrontamiento en el Deporte (ACSQ-1) a 22 nadadores individuales y 28 jugadores de waterpolo, minutos antes de una competición oficial. Los resultados indican que existen diferencias solo en los niveles de autoconfianza, pero que la relación entre estrategias de afrontamiento y ansiedad precompetitiva es distinta entre grupos. Finalmente, se sugieren modelos diferenciados de intervención, el alcance de los resultados, las limitaciones del estudio y se sugieren nuevas investigaciones.
\end{abstract}

Palabras Clave: Deporte, Psicología del Deporte, Ansiedad Precompetitiva y Estrategias de Afrontamiento.

\begin{abstract}
There is extensive evidence of the effects of precompetitive anxiety (e.g. Bueno, Capdevila,\& Fernández-Castro, 2002; Caracuel and Jaenes, 2015) in sports practices and events, as well as the protective role of coping strategies employed by athletes (e.g., Soto, 2003). However, because competitive sports practices differ, among other things, in their collective or individual character, both competitive anxiety and the coping strategies involved could be differentiated according to that condition (e.g., Simon and Martens, 1979). This study corresponds to an exploration of possible differential effects of the type of practice on precompetitive anxiety, coping strategies and the relationship between both. The design was transversal and correlational kind, in which the Inventory of Competitive Anxiety in Sport (CSAI-2) and the Questionnaire of Approach to Facing in Sport (ACSQ-1) were applied to 22 individual swimmers and 28 water polo players, minutes before an official competition. Results indicate that there are differences only in levels of self-confidence, but that the relationship between coping strategies and precompetitive
\end{abstract}

* Autor correspondiente / Corresponding author: geraaldy@gmail.com 
anxiety is different between groups. Finally, differentiated models of intervention, the scope of the results, the limitations of the study, and new research are suggested.

Key Words: Sports, Sports Psychology, Precompetitive Anxiety and Coping Strategie.

\section{INTRODUCCIÓN}

El deporte constituye un fenómeno social que contribuye al desarrollo personal, por medio del progreso en el bienestar físico, psicológico y social, dando lugar a una mejor calidad de vida (Irazusta \& Arruza, 2006; Romero, García-Mas, \& Brustad, 2009). Dentro de la literatura, se han señalado sus diversas contribuciones, tanto para la salud física (Bouchard, Bar-Or, \& Malina, 2004; Britapaz \& Del Valle, 2015) como para la salud mental, disminuyendo el estrés y aumentando el bienestar general (Jiménez, Martínez, Miro, \& Sánchez, 2008). Sin embargo, al igual que las prácticas deportivas impactan en el estado psicológico, existe fuerte evidencia del impacto de factores psicológicos (e.g. ansiedad, nerviosismo, presiones psicológicas) sobre los desempeños en deportes competitivos (Molina, Sandín, \& Chorot, 2014).

El deporte competitivo, se caracteriza por ser una variación de la actividad física, basada en un trabajo programado y riguroso, constituido como un proceso continuo y de permanencia en el tiempo, en base a metas específicas relacionadas con competiciones y resultados (Blázquez, 1995; Moreno, Águila, \& Borgues, 2011). Estas características no solo implican un fuerte compromiso conductual y psicológico con la actividad, sino que son generadoras de intensas emociones, cuando las exigencias aumentan (Dias, Cruz, \& Fonseca, 2011; Muñoz de Morales \& Bisquerra, 2006), las que a su vez, se ven fuertemente influenciadas por otras variables como: la incertidumbre del resultado, el público, las expectativas de personas significativas y la motivación (Bueno, Capdevila, \& FernándezCastro, 2002; Ramis, Torregrosa, Viladrich, \& Cruz, 2013). Si bien todas estas variables tienen un rol fundamental en el desempeño y actuación de los deportistas, una de las variables psicológicas más analizadas en diferentes actividades físicas, es la ansiedad pre-competitiva, considerada como factor influyente en el rendimiento deportivo (Bueno et al., 2002; Pinto \& Vásquez, 2013).
La ansiedad pre-competitiva, es un tipo de ansiedad de estado, que refiere a la reacción emocional de aprehensión, tensión, y nerviosismo ante situaciones de competencia (Dosil, 2008; Martens, Vealy, \& Burton, 1990), la cual puede favorecer o perjudicar el rendimiento de los deportistas (Pineda-Espejel, López-Walle, \& Tomás, 2015). Desde una aproximación multidimensional, la ansiedad pre-competitiva se compone de tres elementos: ansiedad somática; ansiedad cognitiva; y autoconfianza (e.g. Martens et al.,1990; RuizJuan, Zarau, \& Flores-Allende, 2016).

La ansiedad somática, hace referencia al aumento de los indicadores fisiológicos, tales como tensión muscular, aumento de la frecuencia cardiaca, malestares estomacales y la sudoración; la ansiedad cognitiva refiere a la evaluación negativa que realiza el sujeto sobre su rendimiento y resultados (e.g. pensamientos de inseguridad, pérdida total de concentración o disminución de la misma; Navarro Guzmán, Amar, \& González, 1995); y, la autoconfianza, refiere al grado de convicción del deportista de poder lograr lo que se propone, la cual opera como un regulador de la ansiedad (Burton, 1988; Chamberlain \& Hale, 2007; Chirivella \& Esquiva, 2012; Modroño \& Guillén, 2011; Ruiz-Juan et al., 2016).

Si bien el manejo y control de la ansiedad pre-competitiva depende de diversos factores (e.g. autoconfianza, optimismo, relaciones interpersonales; León-Prados, Fuentes, \& Calvo, 2014; Ortín-Montero, De la Vega, \& Gosálvez-Botella, 2013), en la literatura se observa de forma consistente que, cuando los niveles de ansiedad son elevados, se despliegan estrategias de afrontamiento para reducir la ansiedad y restaurar el equilibrio psicológico (Soto, 2003; Dias et al., 2011).

Las estrategias de afrontamiento refieren a los esfuerzos cognitivos y conductuales que se ponen en marcha para manejar las demandas, externas y/o internas, que son evaluadas como mayores a los recursos del individuo. Es decir, son un conjunto de respuestas que elabora el individuo 
ante diversas situaciones estresantes (Lazarus \& Folkman, 1986; Márquez, 2006).

Dada su composición multivariante, en la literatura se distinguen dos grandes tipos de estrategias de afrontamientos, las orientadas hacia el objetivo, donde se pretende cambiar aspectos de la situación a través de la planificación y análisis lógico (e.g. Smith, Ntoumanis, Duda, \&Vansteenkiste, 2011); y las orientadas a la emoción, donde las acciones que se emplean tienen como finalidad la regulación de las emociones, mediante el autocontrol y la búsqueda de apoyo (e.g. Poliseo \& McDonough, 2012).

La adecuada utilización de las estrategias de afrontamiento, durante las competiciones, ayudan a manejar las emociones negativas, organizar la recepción de la información, planificar las respuestas según los recursos disponibles y a la ejecución de las acciones apropiadas, permitiendo obtener un control favorable de la ansiedad, por medio de la resolución del conflicto (Cantón-Chirivella, Checa-Esquiva, \& Vellisca-González, 2015; Pinto \& Vásquez, 2013). Por otra parte, cuando el uso de estas estrategias es inadecuado y/o insuficiente, las emociones negativas, a largo plazo, se traducen en insatisfacción deportiva, empobrecimiento de la ejecución e, inclusive, el abandono de la práctica deportiva (Márquez, 2006). Es por esto que, la utilización de estrategias de afrontamiento efectivas, permite a los deportistas mejorar tanto su rendimiento deportivo como su autoconfianza y autocontrol emocional (Letelier, 2007).

La relación entre ansiedad pre-competitiva y estrategias de afrontamiento, ha sido constatada ampliamente en múltiples disciplinas deportivas, tanto individuales (e.g. tenis, natación, atletismo, golf; Letelier, 2007; Pozo, 2007; Pinto \& Vázquez, 2013; Ruiz-Juan et al., 2016) como colectivas (e.g. futbol, baloncesto, voleibol; Abian Vicen, Báguena, \& Abian, 2015; De la Vega, Ruiz, García, \& Del Valle, 2011; León-Prados et al., 2014; Morillo, Reigal, \& Hernández-Mendo, 2016). Sin embargo, la responsabilidad, la confianza y apoyo social puede diferir entre ambos tipos de prácticas, existiendo la posibilidad que, tanto la ansiedad pre-competitiva como las estrategias de afrontamiento involucradas, se desplieguen de modo diferencial entre ambos tipos de prácticas (Días, Cruz, \& Fonseca, 2011).

Si bien la investigación en base a la ansiedad pre-competitiva y las estrategias de afrontamientos en deportistas no es un fenómeno emergente (Navarro Guzmán et al., 1995; Urra, 2014; Días et al., 2011), la mayoría de los estudios, son en poblaciones extranjeras, (Ruiz-Juan et al., 2016; Morillo et al., 2016), evidenciándose un baja presencia de estudios en el contexto latinoamericano, particularmente en lo referido a posibles funcionamientos diferenciales de ansiedad pre-competitiva y las estrategias de afrontamiento entre disciplinas individuales y colectivas.

Por tanto, el objetivo del presente estudio es comparar la relación de estrategias de afrontamiento con ansiedad pre-competitiva, en un contexto sudamericano. Entre deportistas que practican waterpolo y natación, disciplinas realizadas en un mismo medio y con entrenamientos parcialmente compartidos, pero que difieren, entre otras cosas, en el carácter colectivo o individual de las competiciones.

\section{MÉTODO}

\section{Diseño y participantes}

Diseño transversal con encuestas de alcance correlacional. El Muestreo fue no probabilístico y por disponibilidad. La muestra total fue de 50 personas, estudiantes de educación media (i.e. equivalente a educación secundaria), de nacionalidad chilena y sexo masculino. Los participantes de deportes colectivos fueron 28 waterpolistas, cuyas edades fluctúan entre los 12 y 17 años (DT $=1,7$ ), quienes compiten en la categoría sub-15 o sub-18; los participantes de deportes individuales se fueron 22 nadadores competitivos cuyas edades fluctúan entre los 12 a los $17(\mathrm{DT}=3,1)$ años.

\section{Instrumentos}

Datos sociodemográficos, en este apartado se recogió información acerca del sexo, edad, nacionalidad, nivel del estudio de los encuestados. Inventario de Ansiedad Competitiva en el Deporte (Competitive Sport Anxiety Inventory, CSAI-2), desarrollada inicialmente por Martens Burton, Vealey, Bump y Smith (1990) y traducida al castellano por Capdevila (1997). Este inventario, tiene como propósito evaluar tres dimensiones presentes en la ejecución deportiva (i.e. ansiedad cognitiva, somática y autoconfianza), mediante 27 ítems, con formato tipo Likert de 4 alternativas, donde 1 corresponde a "nada" y 4 corresponde a "mucho". Existen evidencia de validez, basada en la estructura interna del test, y fiabilidad adecuados 
(i.e. $\geq 0,78$ ), en población hispanohablante (Andrade, Lois, \& Arce, 2007).

Cuestionario de Aproximación al Afrontamiento en el Deporte (Approach to Coping in Sport Questionnaire, ACSQ-1), desarrollada por Kim y Duda (1997) y traducida al castellano por Kim, Duda, Tomás y Balaguer (2003). Este cuestionario mide 5 dimensiones (i.e. Calma Emocional, referida a los intentos por controlar las emociones negativas a través del manejo de la técnica, los pensamientos y los síntomas físicos; Planificación activa/Reestructuración cognitiva, referida a los intentos por resignificar una situación adversa, organizando las propias acciones y los pensamientos para conseguir un objetivo específico; Retraimiento mental, pensamientos de aceptación frente a la incapacidad de conseguir un objetivo; Conductas de riesgo, utilización de habilidades, estrategias y técnicas complejas que van más allá de los niveles actuales del rendimiento; y Búsqueda de apoyo social, alude a la necesidad del deportista de estrechar sus relaciones interpersonales en momentos difíciles), mediante 28 ítems, con formato Likert de 5 puntos, donde 1 toma el valor de "nunca" y 5 el valor de "siempre". Esta escala cuenta con evidencias de validez basada en la estructura interna del test y en la relación con otras variables, así como adecuados niveles de fiabilidad $(\alpha>0,7)$ (Kim et al., 2003).

\section{Procedimiento}

En una primera instancia, se obtuvieron las autorizaciones de los entrenadores de los clubes de natación y waterpolo de la ciudad de Arica. Acordada la participación, se procedió al acercamiento con los jóvenes, entregándoles un consentimiento informado a los deportistas que otorgaron su asentimiento informado, bajo el compromiso de confidencialidad y participación voluntaria, se les aplico los instrumentos del estudio una hora antes de la competencia.

\section{Análisis de datos}

Primeramente, se realizaron los análisis descriptivos de las variables ansiedad pre-competitiva y estrategias de afrontamiento. Para evaluar la diferencia entre las medias observadas en los grupos, dado que se sostuvo el supuesto de normalidad para todas las variables $(\mathrm{pKS}>0,05)$ en ambos grupos, se utilizó la prueba $\mathrm{T}$ de Student para muestras independientes. Finalmente, para analizar la relación entre ansiedad pre-competitiva y estrategias de afrontamiento, dado el supuesto de normalidad y el carácter continuo de las variables, se realizaron análisis de correlaciones de Pearson. Todos los análisis son ejecutados con el programa IBM SPSS Statistics en su versión 24.

\section{Resultados}

En la tabla 1 se presenta los estadísticos descriptivos, medias y desviaciones típicas de ansiedad precompetitiva y estrategias de afrontamiento, diferenciados por grupos (i.e. deporte individual y deporte colectivo). En ambos grupos, se observan valores medios (aprox. 3), en las dimensiones de estrategias de afrontamiento, con la salvedad de calma emocional y reestructuración cognitiva, cuyos valores son medio-alto (aprox. 4). En el caso de ansiedad precompetitiva, se observa que los valores tienden a ser medios-bajos (aprox. 2), salvo en autoconfianza, en la cual presenta valores medios (aprox. 3).

La tabla 2, presenta los análisis de diferencias de medias entre Ansiedad pre-competitiva y Estrategias de afrontamiento. A través de la prueba T Student, para muestras independientes.

Tanto en las dimensiones de estrategias de afrontamiento como ansiedad precompetitiva no se observaron diferencias poblacionales entre el grupo de natación y el de waterpolo ( $\mathrm{p}>.05$ ). En el caso de la subdimensión autoconfianza, se estima que el grupo de práctica colectiva posee una autoconfianza media entre .72 y .13 puntos mayor a los de práctica individual.

En la tabla 3, se observan las matrices de correlaciones de Pearson, diferenciadas por grupos. Se observa que existen diferencias aparentes en las relaciones entre las estrategias de afrontamiento y la ansiedad pre-competitiva, según el tipo de práctica deportiva. En el caso de la natación, se observaron relaciones moderadas, y estadísticamente distintas de cero, entre: ansiedad y calma emocional ( $\mathrm{r}=$ $.478 ; \mathrm{p}<, 000)$; ansiedad y reestructuración cognitiva $(\mathrm{r}=.440 ; \mathrm{p}<.000)$; así como una relación inversa moderada de ansiedad con retraimiento mental $(\mathrm{r}=-.494 ; \mathrm{p}<.000)$. Respecto a las relaciones en el grupo de waterpolo, sólo se observa un efecto moderado y estadísticamente significativo en la relación entre conductas de riesgo y autoconfianza $(\mathrm{r}=.434 ; \mathrm{p}<.000)$. Adicionalmente, si bien se observan otros efectos moderados, debido al reducido tamaño muestral, no es sostenible afirmar dichas relaciones a nivel poblacional. 
Tabla 1. Estadísticos descriptivos, por tipo de disciplina natación y waterpolo.

\begin{tabular}{|c|c|c|c|c|c|}
\hline Deporte & & Mínimo & Máximo & Media & DT \\
\hline \multirow{8}{*}{ Natación } & Calma emocional & 2.43 & 5.00 & 4.04 & .69 \\
\hline & $\begin{array}{l}\text { Reestructuración } \\
\text { cognitiva }\end{array}$ & 2.00 & 5.00 & 3.96 & .79 \\
\hline & Retraimiento mental & 1.00 & 3.67 & 1.97 & .67 \\
\hline & $\begin{array}{l}\text { Conductas } \\
\text { de riesgo }\end{array}$ & 1.00 & 4.33 & 3.11 & .90 \\
\hline & $\begin{array}{l}\text { Búsqueda de apoyo } \\
\text { social }\end{array}$ & 1.00 & 5.00 & 3.02 & 1.26 \\
\hline & Ansiedad cognitiva & 1.33 & 3.78 & 2.30 & .70 \\
\hline & Autoconfianza & 1.67 & 3.78 & 2.94 & .58 \\
\hline & Ansiedad somática & 1.00 & 3.33 & 1.94 & .64 \\
\hline \multirow{8}{*}{ Waterpolo } & Calma emocional & 2.86 & 5.00 & 4.04 & .57 \\
\hline & $\begin{array}{l}\text { Reestructuración } \\
\text { cognitiva }\end{array}$ & 3.00 & 4.67 & 3.81 & .47 \\
\hline & Retraimiento mental & 1.00 & 2.33 & 1.71 & .42 \\
\hline & $\begin{array}{l}\text { Conductas } \\
\text { de riesgo }\end{array}$ & 2.00 & 4.75 & 3.36 & .79 \\
\hline & $\begin{array}{l}\text { Búsqueda de apoyo } \\
\text { social }\end{array}$ & 1.40 & 4.80 & 2.97 & .87 \\
\hline & Ansiedad cognitiva & 1.56 & 3.67 & 2.49 & .52 \\
\hline & Autoconfianza & 2.33 & 4.00 & 3.37 & .40 \\
\hline & Ansiedad somática & 1.00 & 2.78 & 1.81 & .48 \\
\hline
\end{tabular}


Tabla 2. Comparación de medias, entre los grupos de natación y waterpolo.

\begin{tabular}{lccccc}
\hline & $t$ & $g l$ & $p$ & \multicolumn{2}{c}{$\begin{array}{c}\text { Intervalo de confianza } \\
\text { para la diferencia }\end{array}$} \\
\hline Calma emocional & .02 & 49 & .988 & -.35 & .36 \\
Reestructuración cognitiva & .85 & 49 & .398 & -.20 & .51 \\
Retraimiento mental & 1.68 & 49 & .100 & -.05 & .56 \\
Conductas de riesgo & -1.03 & 49 & .310 & -.72 & .23 \\
Búsqueda de apoyo social & .18 & 35 & .862 & -.58 & .69 \\
Ansiedad cognitiva & -1.06 & 49 & .294 & -.52 & .16 \\
Ansiedad somática & .82 & 49 & .414 & -.18 & .44 \\
Autoconfianza & -2.94 & 35 & .006 & -.72 & -.13 \\
\hline
\end{tabular}

Nota: $t$ : valor del estadístico de t de Student de grupos independientes; $g l$ : grados de libertad; $p$ : nivel de significación $(p<.05)$

Tabla 3. Matriz de correlaciones de Pearson, diferenciadas por grupo de natación y waterpolo.

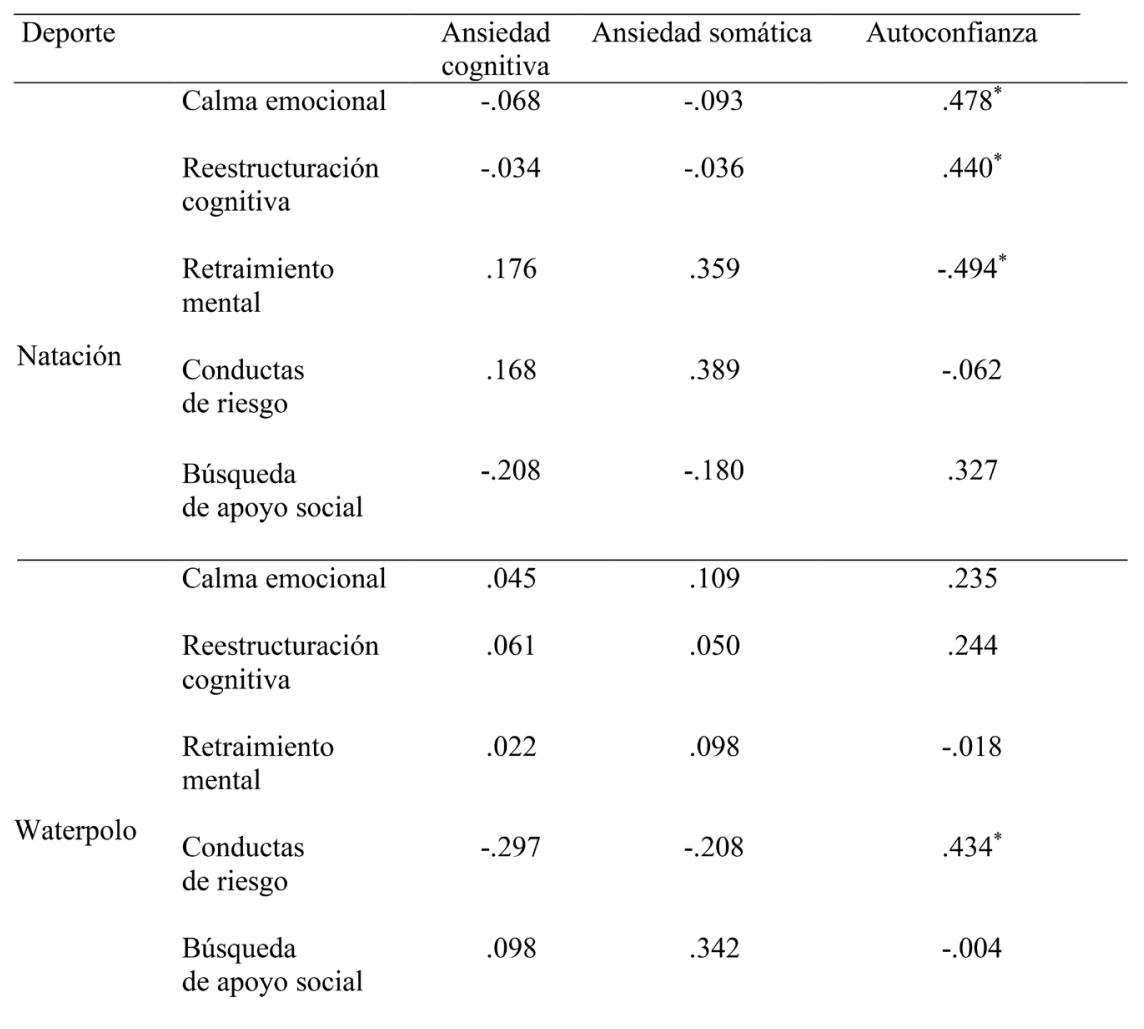

Nota: la correlación es significativa al nivel $* \mathrm{p}<.05$ 


\section{DISCUSIÓN}

Aún cuando en la literatura se han señalado efectos diferenciales en los niveles de ansiedad precompetitiva y estrategias de afrontamiento, entre diferentes tipos de deportes (e.g. Días et al., 2010; Mellalieu, Hanton, \& O'Brien, 2004), los datos reportados no permiten sostener, en términos generales, la existencia de diferencias atribuibles al carácter individual o colectivo de la práctica, ya que se observan medias equivalentes entre ambos grupos. Sin embargo, en la subdimensión autoconfianza se observa un nivel medio mayor en prácticas colectivas.

Esta discrepancia específica, concuerda con otros estudios, donde el deporte en equipo ha evidenciado propiciar una mayor confianza en los miembros en comparación a las prácticas individuales (Molina, Oriol, \& Mendoza, 2017; Cantón-Chirivella et al., 2015; Pinto \& Vásquez, 2013), lo que podría suponer que al favorecer la autoconfianza, las prácticas colectivas incrementarían indirectamente el rendimiento y el estado emocional positivo de los deportistas (e.g. Feltz, 2007; Martínez-Romero, Molina, \& Oriol- Granado, 2016), teniendo el potencial de disminuir la ansiedad cognitiva y somática (Cervantes, Rodas, \& Capdevila, 2009; Prados, García, \& Lluch, 2011).

En lo que refiere a la relación entre ansiedad precompetitiva y estrategias de afrontamiento, tanto en la práctica individual (natación) como en la práctica colectiva (waterpolo), no se observan efectos de ninguna de las estrategias de afrontamiento sobre ansiedad cognitiva y ansiedad somática, no obstante, se observan que algunas estrategias de afrontamiento poseen efectos diferenciales sobre la autoconfianza.

En el caso de la natación se reportan efectos de las estrategias de calma emocional, reestructuración cognitiva y retraimiento mental, respecto a las prácticas colectivas se observan efectos solo en el caso de conductas de riesgo. Estas diferencias podrían explicarse, por el tipo de entrenamiento que se realiza en las prácticas individuales, donde el tiempo y esfuerzo que dedican depende de sí mismos, lo que contribuye al uso prioritario de la estabilidad emocional y la concentración (Ruiz-Juan et al., 2016; Morillo et al., 2016), como estrategias para incrementar los niveles de autoconfianza (Pinto, 2011). En este mismo sentido, en un estudio realizado por Moreno et al. (2017) se identificaron estas estrategias de afrontamientos en nadadores de alto rendimiento, enfatizando en que los deportistas presentan estrés precompetitivo, pero que lo disminuyen, a través de pensamientos positivos y búsqueda de soluciones al igual que los nadadores de este estudio. En cambio, los deportistas de prácticas colectivas (waterpolo), tenderían a utilizar conductas de riesgo como estrategia para aumentar su autoconfianza, es decir, realizar acciones que van más allá de los niveles actuales de su rendimiento (Romero et al., 2010), esta relación concuerda otros trabajos investigativos, las cuales han centrado sus análisis en la percepción del éxito (autoeficacia) $y$ el rendimiento real en una competencia, como una relación negativa (Vancouver, Thompson, Tischner, \& Putka, 2002), donde se cuestionaría la capacidad auto regulatoria de los deportistas.

$\mathrm{Si}$ bien los resultados muestran diferencias menores en ansiedad precompetitiva y estrategias de afrontamiento entre deportistas de waterpolo y natación, los efectos diferenciales observados, como aproximación exploratoria, sugiere la necesidad de dar un énfasis diferencial al tipo de afrontamiento según el carácter individual o colectivo de las disciplinas.

En líneas de investigación futura, se sugiere replicar este estudio con otras prácticas equivalentes de corte individual y grupal, incrementando los tamaños muestrales. Como también, se hace necesario indagar de qué manera la autoconfianza puede favorecer la frecuencia con que son utilizadas las estrategias de afrontamiento durante un partido, o cómo la preparación técnica puede ayudar a constituir un perfil de afrontamiento en los deportistas de prácticas individuales y colectivas.

El presente estudio sugiere considerar el tipo de práctica deportiva en los modelos de intervención de la psicología del deporte para mejorar el rendimiento deportivo, diferenciando el abordaje de prácticas individuales y colectivas.

\section{LIMITACIONES}

Si bien, los hallazgos del presente estudio han aportado evidencia respecto a las relaciones entre la ansiedad precompetitiva y las estrategias de afrontamiento en disciplinas individuales y colectivas, sus conclusiones, deben ser consideradas con precaución, entendiéndolas como exploratorias, debido al tamaño muestral. 


\section{CONCLUSIONES}

El presente estudio, examinó la relación de ansiedad pre-competitiva y estrategias de afrontamiento, en deportistas de waterpolo y natación, en un contexto sudamericano.

Los resultados demuestran que no hay diferencias entre el carácter colectivo o individual del deporte, excepto en autoconfianza donde el waterpolo obtiene un puntaje superior a quienes practican la natación. Al analizar la relación de ansiedad pre-competitiva y estrategias de afrontamiento, en ambos casos se aprecian relaciones significativas con autoconfianza, pero se observan diferencias en algunas relaciones, ya que, en el caso de natación, las estrategias de afrontamiento con impactos sobre la ansiedad precompetitiva son calma emocional, reestructuración cognitiva y retraimiento mental, mientras que en deportes colectivos solo posee efectos estadísticamente significativos la estrategia de conductas de riesgo. 


\section{Referencias}

Abian Vicen, J., Báguena Mainar, J. I., \& Abian Vicen, P. (2015). Estrés pre-competitivo en jugadores adolescentes de bádminton. Psicología del Deporte, 24(2), 217-224.

Andrade, E.M., Lois, G., \& Arce, C. (2007). Propiedades psicométricas de la versión española del Inventario de Ansiedad Competitiva CSAI-2R en deportistas. Psicothema, 19(1), 150-155.

Blázquez, D. (1995). La iniciación deportiva y el deporte escolar. Barcelona: Inde.

Britapaz, L. \& Del Valle, J. (2015). Significado del deporte en la dimensión social de la salud. Salus, 19, 28-33.

Bueno, J., Capdevila, L., \& Fernández- Castro, J. (2002). Sufrimiento competitivo y rendimiento en deportes de resistencia. Revista de Psicología del deporte, 11(2), 209-226.

Burton, D. (1988). Do anxious swimmers swim slower? Reexamining the elusive anxiety-performance relationship. Journal of Sport \& Exercise Psychology,10(1), 45-61.

Cantón-Chirivella, E., Checa-Esquiva, I., \& Vellisca-González, M. (2015). Bienestar psicológico y ansiedad competitiva: el papel de las estrategias de afrontamiento. Revista Costarricense de Psicología, 34(2). Recuperado de: http://www.rcps-cr.org/ openjournal/index.php/RCPs/article/view/66

Cervantes, J. C., Rodas, G., \& Capdevila, L. (2009). Perfil psicofisiológico de rendimiento en nadadores basado en la variabilidad de la frecuencia cardíaca y en estados de ansiedad precompetitiva. Revista de psicología del deporte, 18(1), 37-52.

Chamberlain, S. T. y Hale B. D. (2007). Competitive state anxiety and self confidence: Intensity and direction as relative predictors of performance on a golf putting task. Anxiety, Stress, and Coping, 20(2), 197-207.

Chirivella, E. C. \& Esquiva, I. C. (2012). Los estados emocionales y su relación con las atribuciones y las expectativas de autoeficacia en el deporte. Revista de Psicología del Deporte, 21(1), 171-176.

De la Vega, R., Ruiz, R., García, G., \& Del Valle, S. (2011). El estado de ánimo precompetitivo en un equipo de fútbol profesional: Un estudio entre jugadores titulares y suplentes. Cuadernos de psicología del deporte, 11(2), 107-117.

Dias, C. S., Cruz, J. F. A., \& Fonseca, A. M. (2011). Ansiedad, percepción de amenaza y estrategias de afrontamiento en el deporte: diferencias individuales en el rasgo de ansiedad. Ansiedad y estrés, 17(1), 1-13. Recuperado de: http://hdl. handle.net/1822/15278

Dosil, J. (2008). Psicologia de la Actividad Física y del Deporte. Madrid: McGraw Hill. doi: https://doi. org/10.4272/978-84-9745-351-6

Feltz, D. L. (2007). Self-Confidence and Sports Performance. Studies, 33(41), 50-66. doi: http://dx.doi. org/10.1249/00003677-19880016000016

Irazusta, S. \& Arruza, J. (2006). Influencia de variables psicológicas en el rendimiento de jugadoresamateurs de golf. Revista de Psicología del Deporte, 15(1), 127-138.

Jiménez, M.G., Martínez, P., Miró, E., \& Sánchez, A.I. (2008). Bienestar psicológico y hábitos saludables: ¿están asociados a la práctica del ejercicio? International Journal of Clinical Health Psychology, 8(1), 185-202.

Kim, M. S., Duda, J. L., Tomás, I., \& Balaguer, I. (2003). Examination of the psychometric properties of the spanish version of the approach to coping in sport questionnaire. Revista de Psicología del Deporte, 12(2), 197-212.

Lazarus, R. S. y Folkman, S. (1986). Estrés y procesos Cognitivos. Barcelona: Martínez-Roca.

León-Prados, J., Fuentes, I., \& Calvo, A. (2014). Relación entre ansiedad estado, autoconfianza percibida y rendimiento en baloncesto. Revista internacional de medicina y ciencias de la actividad física y el deporte, 14(55),527-543.

Letelier, A. (2007). Estudio correlacional entre la ansiedad estado competitiva y las estrategias de afrontamiento deportivo en tenistas juveniles. [Memoria para optar a título de Psicóloga]. Universidad de Chile, Facultad de Ciencias Sociales, Santiago.

Márquez, S. (2006). Estrategias de afrontamiento del estrés en el ámbito deportivo: fundamentos teóricos e instrumentos de evaluación. International Journal of Clinical and Health Psychology, 6(2), 359-378.

Martens, R., Vealey, R. S., \& Burton, D. (1990). Competitive anxiety in sport. United Stage, Champaign, IL: Human Kinetics.

Martínez-Romero, M., Molina, V.M., \& Oriol-Granado, X. (2016). Desarrollo y validación del cuestionario de autoconfianza en competición CACD para deportes individuales y colectivos. Cuadernos de Psicología del Deporte, 16(2), 13-20.

Mellalieu, S. D., Hanton, S., \& O'Brien, M. (2004). Intensity and direction of competitive anxiety as a function of sport type and experience. Scandinavian Journal of Science and Medicine in Sport, 14(5), 326-334. doi: 10.1111/j.1600-0838.2004.00389.x

Modroño, C. \& Guillén, F. (2011). Anxiety Characteristics of Competitive Windsurfers: Relationships with Age, Gender, and Performance Outcomes. Journal of Sport Behavior, 34(3), 281-294.

Molina, J., Sandín, B., \& Chorot, P. (2014). Sensibilidad la ansiedad y presión psicológica: Efectos sobre el rendimiento deportivo en adolescentes. Cuadernos de Psicología del Deporte, 14(1), 45-54.

Molina, V., Oriol, X., \& Mendoza, M. (2017). Emotional regulation and physical recovery in young athletes of individual and collective sport modalities. Revista Internacional De Ciencias Del Deporte, 14(53), 191-204. doi: https://doi.org/10.5232/ ricyde2018.05301

Moreno, J.A., Águila, C., \& Borges, F. (2011). La socialización en la práctica físico deportiva de carácter recreativo: predictores de los motivos sociales. Apunts. Educación Física y Deportes, 103(1), 76-82.

Moreno, P. J. F., Ruvalcaba, S. L. P., Salazar, C. M., Lozano, E. G. M., Gutiérrez, C. J. L., Pineda, L. T. B., \& Mendoza, E. J. M. (2017). Fuentes, síntomas y estrategias de afrontamiento al estrés-competitivo en nadadores. Revista de psicología del deporte, 26(2), 199-209.

Morillo, J. P., Reigal, G. E., \& Hernández-Mendo, A. (2016). Relaciones entre el perfil psicológico deportivo y la ansiedad competitiva en jugadores de balonmano playa. Revista de Psicología del Deporte, 25(1), 121-128.

Muñoz de Morales, M. \& Bisquerra R. (2006). Evaluación de un programa de educación emocional para la prevención del estrés psicosocial en el contexto del aula. Ansiedad y Estrés, $12,401-412$.

Navarro Guzmán, J. I., Amar, J. R., \& González Ferreras, C. (1995). Ansiedad pre-competitiva y conductas de autocontrol 
en jugadores de fútbol. Revista de psicología del deporte, 4(2), 0007-017.

Pineda-Espejel, A., López-Walle, J., \& Tomás, I. (2015). Factores situacionales y disposicionales como predictores de la ansiedad y autoconfianza precompetitiva en deportistas universitarios. Cuadernos de Psicología del Deporte, 15(2), 55-69. Recuperado de https://search-proquest-com.ezproxy.biblio.uta. $\mathrm{cl} /$ docview $/ 1702273247$ ?accountid $=44468$

Pinto, M. F. \& Vásquez, N. (2013). Ansiedad estado competitiva y estrategias de afrontamiento: su relación con el rendimiento en una muestra argentina de jugadores amateurs de golf. Revista de Psicología del Deporte, 22(1), 47-52.

Pinto, M. F. (2011). Análisis de la relación entre el uso de estrategias de afrontamiento, el posicionamiento final en torneo y el hándicap de jugadores amateurs de golf. III congreso internacional y práctica profesional en psicología. Simposio llevado a cabo en la XVIII jornada de investigación del séptimo encuentro de investigadores en psicología de MERCOSUR en la Universidad de Buenos Aires. Buenos Aires, Argentina.

Poliseo, J. M. \& McDonough, M. H. (2012). Coping effectiveness in competitive sport: Linking goodness of fit and coping outcomes. Sport, Exercise, and Performance Psychology, 1(2), 106-119. doi: http://dx.doi.org/10.1037/a0026382

Pozo, A. (2007). Intensidad y dirección de la ansiedad competitiva y expectativas de resultados en atletas y nadadores. Revista de Psicología del Deporte, 16(2), 137-150.

Prados, J. A. L., García, I. F., \& Lluch, Á. C. (2011). Ansiedad estado y autoconfianza precompetitiva en gimnastas. RICYDE. Revista Internacional de Ciencias del Deporte, 7(23), 76-91.

Ramis, Y., Torregrosa, M., Viladrich, C., \& Cruz, J. (2013). El apoyo a la autonomía generado por entrenadores, compañeros y padres y su efecto sobre la motivación autodeterminada de deportistas de iniciación. Anales de Psicología, 29(1), 243-248. doi: http://dx.doi.org/10.6018/analesps.29.1.124011

Robert, M. (2004). Growth, Maturation, and Physical Activity. Champaign, IL: Human Kinetics.
Romero, A. E., Zapata, R., García-Mas, A., Brustad, R. J., Garrido, R., \& Letelier, A. (2010). Estrategias de afrontamiento y bienestar psicológico en jóvenes tenistas de competición. Revista de Psicología del Deporte, 19(1), 117-133.

Romero, A. E., García-Mas, A., \& Brustad, R. J. (2009). Estado del arte, y perspectiva actual del concepto de bienestar psicológico en psicología del deporte. Revista Latinoamericana de Psicología, 41(2), 335-347.

Ruiz-Juan, F. \& Zarauz, A. (2013). Factores determinantes de la ansiedad en atletas veteranos españoles. Universitas Psychologica, 13(3),10-48.

Ruiz-Juan, F., Zarauz Sancho, A., \& Flores-Allende, G. (2016). Ansiedad precompetitiva en corredores de fondo en ruta en función de sus variables de entrenamiento (Precompetitive anxiety in long-distance runners depending on their training variables). Retos, 0(30), 110-113. Recuperado de https://recyt. fecyt.es/index.php/retos/article/view/43674

Smith, A.L, Ntoumanis, N., Duda, J.L., \& Vansteenkiste, M. (2011). Goal Striving, Coping, and Well-Being: A Prospective Investigation of the Self-Concordance Model in Sport. Journal of Sport \& Exercise Psychology, 33, 124-145.

Soto, J. (2003). Estudio explicativo del nivel de expectativas de autoeficacia, ansiedad/estado competitiva y ansiedad/ rasgo competitivo en seleccionados chilenos de voleibol categoría menores (sub-17). [Memoria para optar al título de Psicólogo]. Universidad de Chile, Facultad de Psicología, Santiago.

Urra Tobar, B. (2014). Evaluación de la efectividad del entrenamiento de estrategias de afrontamiento en el nivel de ansiedad precompetitiva en tenimesistas. Revista de Psicología del Deporte, 23(1), 67-74.

Vancouver, J., Thompson, C., Tischner, E., \& Putka, D. (2002). Two studies examining the negative effect of self-efficacy on performance. Journal of Applied Psychology, 87(3), 506-516. doi:10.1037/0021-9010.87.3.506 